\title{
Anterior transclavicular approach for lung cancer invading the superior sulcus
}

Kohei Hashimoto, MD, PhD, ${ }^{\mathrm{a}, \mathrm{b}}$ and Hiroyuki Sakamaki, MD, ${ }^{\mathrm{a}}$ Tochigi and Tokyo, Japan

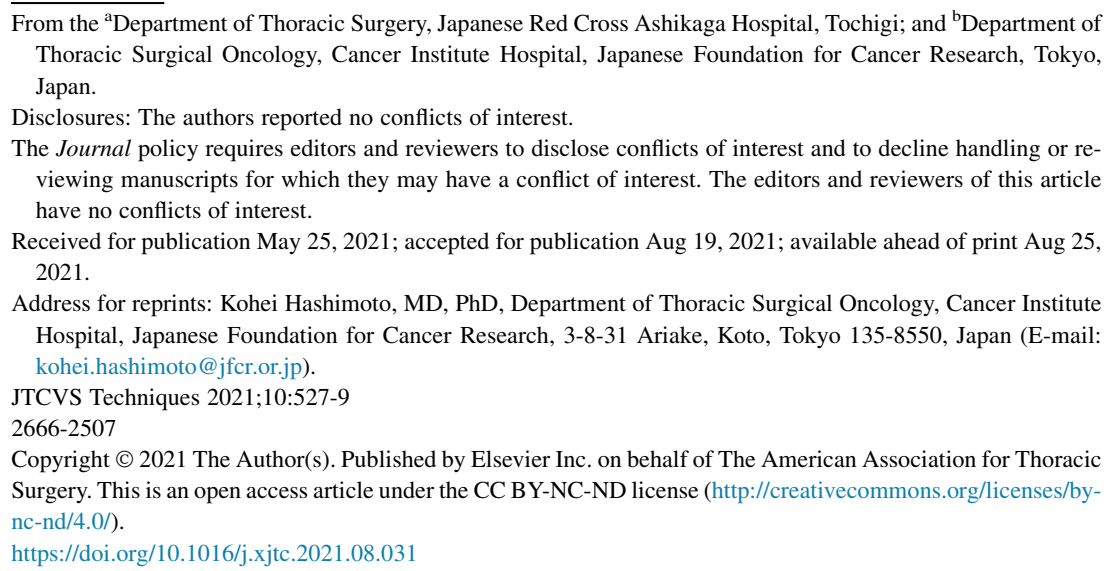

- Video clip is available online.

Complete surgical resection following chemoradiation is recommended in patients with resectable non-small cell lung cancer that invades the superior sulcus. Several approaches have been proposed based on the site of invasion. ${ }^{1}$ We demonstrate a case who underwent anterior transclavicular approach (the Dartevelle approach) for non-small cell lung cancer invading the anatomical territory in the vicinity of the sternoclavicular joint (Video 1). Institutional review board approval was waived, owing to the single case report nature, individual informed consent was not required.

\section{CLINICAL SUMMARY}

A 65-year-old male ex-smoker presented with left shoulder pain and abnormalities on chest radiography, and computed tomography revealed a left apical mass $(4 \mathrm{~cm})$. He had history of tracheostomy due to purulent tonsillitis 10 years ago. His pulmonary function was normal (forced expiratory volume in 1 second $105 \%$; diffusing capacity for carbon monoxide 121\%). A transbronchial biopsy specimen revealed squamous cell carcinoma. A fluorine 18-fluorodeoxyglucose-positron emission tomography did not show any nodal or distant metastases. The results of an endobronchial ultrasound-guided biopsy of the

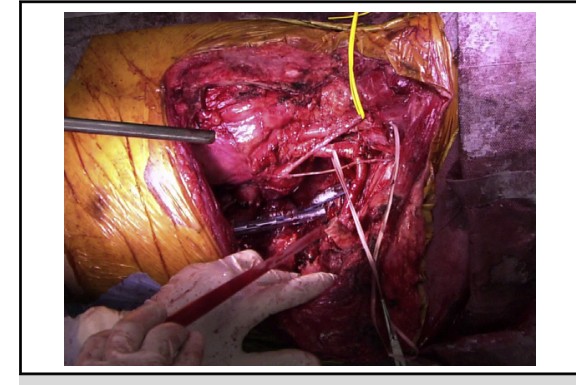

Anterior transclavicular approach allows for excellent exposure of the thoracic inlet.

\section{CENTRAL MESSAGE}

Anterior transclavicular approach facilitates resection of lung cancer close to the sternoclavicular joint. Lobectomy should be performed through a single thoracotomy to preserve shoulder motion.

See Commentaries on pages 530 and 532.

mediastinal lymph nodes (station 4R and 7 were sampled; $4 \mathrm{~L}$ was not amenable to biopsy due to its small size), as invasive mediastinal staging, were negative for malignancy. We observed tumor invasion of the left subclavian vein and the sternal attachment of the first rib and anterior aspect of the second rib (cT4NOM0, Figure 1, A). Magnetic

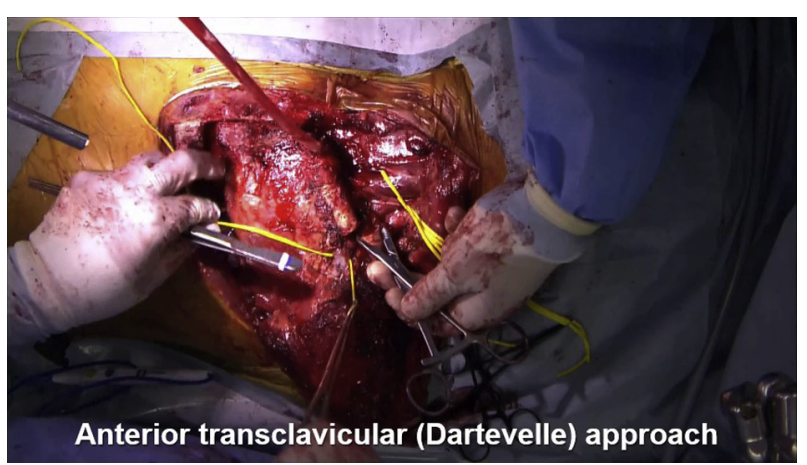

VIDEO 1. Anterior transclavicular (Dartevelle) approach for lung cancer invading the superior sulcus. Video available at: https://www.jtcvs.org/ article/S2666-2507(21)00590-3/fulltext. 


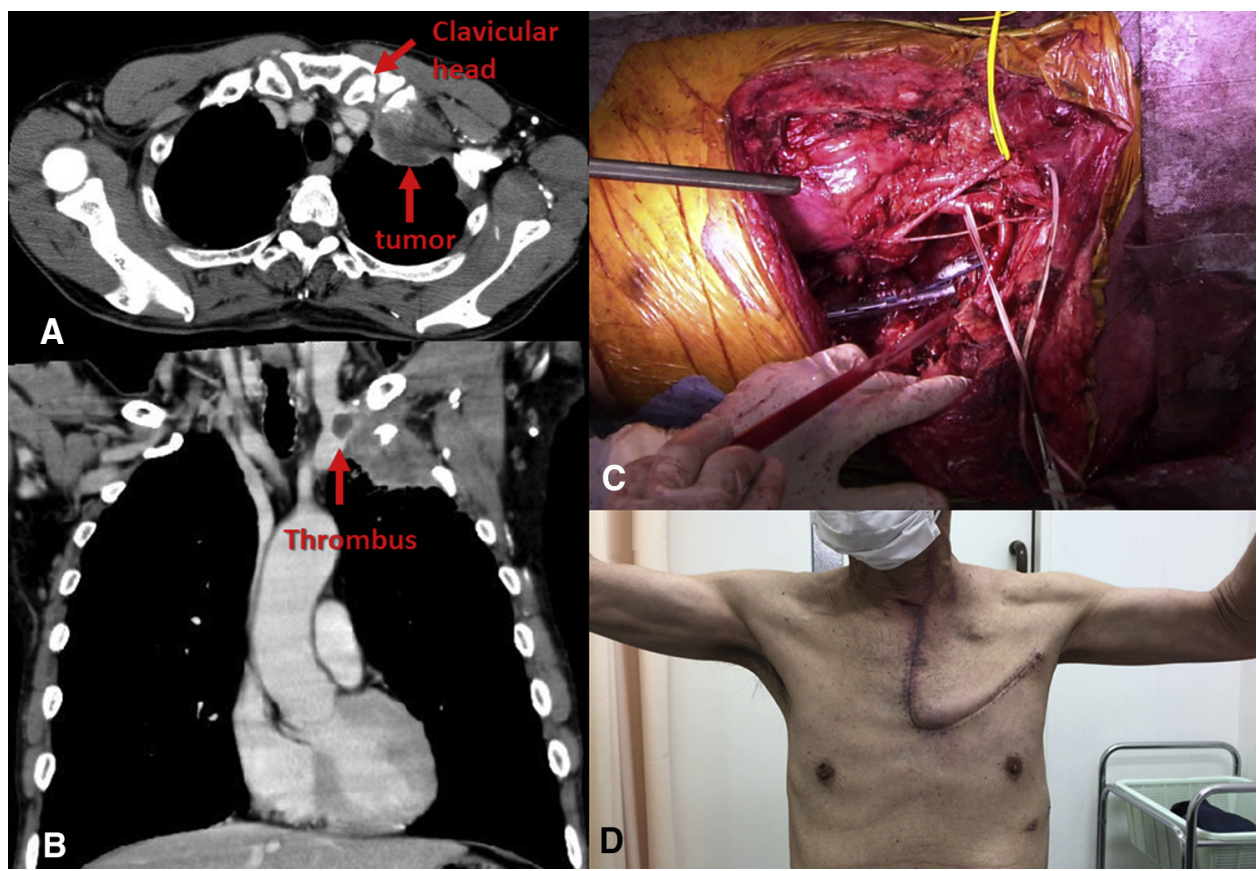

FIGURE 1. Images of a patient undergoing anterior transclavicular approach for a squamous cell carcinoma invading the superior sulcus. A, A preoperative axial image of computed tomography (CT) of the chest showing the tumor invading close to the sternoclavicular joint. B, A preoperative coronal image of $\mathrm{CT}$ of the chest indicating the thrombus in the left subclavian vein adjacent to the tumor. C, An intraoperative image after resection of the chest wall as well as the left upper lobe. D, A view of the shoulder motion 1 month after surgery.

resonance imaging confirmed the invasion of the chest wall and the vein, but it did not show apparent invasion to the left subclavian artery nor the brachial plexus. We also detected a left subclavian vein thrombus (Figure 1, B) that was not evident on computed tomography at the time of presentation (3-week interval).

This venous thrombus that rapidly emerged adjacent to (and proximal to) the tumor was discussed with the cardiology department. Considering the thrombus' rate of growth and that the tumor was the source of the thrombus, we estimated that the risk of pulmonary thrombosis during standard induction therapy was significant even on anticoagulation therapy. We also determined that the chance of a complete resection was highly likely at that point. Therefore, we were afraid that a thrombotic event during induction therapy could cost us the opportunity of performing the curative surgical resection. After the multidisciplinary conference, we decided to perform upfront surgery. We selected the anterior transclavicular approach based on the site of invasion.

The patient was positioned supine, and an incision was made along the left sternocleidomastoid muscle extending to the upper border of the left third rib. A myocutaneous flap was elevated, and thoracotomy was performed through the second intercostal space. Palpation confirmed tumor invasion of the costosternal junction of the first rib. Sternal resection was performed in an "L-shaped" manner from the sternal notch to the second intercostal space. The third rib was also partially resected. The clavicle was cut at the point where the subclavian vein crossed it. The left internal jugular, left subclavian, and left innominate veins were transected. Subclavian artery invasion was not observed. The phrenic nerve was encased and resected. It was subsequently reconstructed using an intercostal nerve graft (although its long-term functionality is unclear as this was not monitored postoperatively). Left upper lobectomy and systematic lymph node dissection were performed through the chest wall defect (Figure 1,C). The chest wall was reconstructed with a Gore-Tex patch (thickness $1 \mathrm{~mm}$; W. L. Gore \& Associates, Inc, Flagstaff, Ariz) and interrupted 2-0 polypropylene sutures. The patch was then covered with the elevated flap. Postoperative chylothorax was treated using a conservative approach.

The patient was discharged on the 18th postoperative day. Histopathologic examination of the resected specimen revealed squamous cell carcinoma, pT4N0M0 stage 3A, with negative margins. The patient underwent adjuvant chemoradiation (4 cycles of platinum-doublet) to complete multimodality therapy. Postoperatively, a $90^{\circ}$ abduction of the left shoulder joint was preserved (Figure 1,D).

\section{DISCUSSION}

The anterior transclavicular approach, originally described by Dartevelle and colleagues ${ }^{2}$ in 1993, provides 
excellent exposure of the thoracic inlet. However, this approach results in some degree of impaired shoulder motion. The transmanubrial osteomuscular-sparing approach, which preserves the sternoclavicular joint and the sternocleidomastoid muscle, was reported to overcome this limitation. ${ }^{3}$ Although preservation of shoulder motion is generally better with the transmanubrial approach than the anterior transclavicular approach, the limitation of shoulder motion in the anterior transclavicular approach can be alleviated by preserving scapulothoracic articulation. ${ }^{4}$ This can be achieved by avoiding additional thoracotomy whenever possible or muscle-sparing thoracotomy.

In fact, the original description of the anterior transclavicular approach, which combined the anterior thoracotomy with posterolateral thoracotomy, was subsequently modified; lobectomy is performed through a single anterior thoracotomy. ${ }^{5}$ Also, de Perrot and Rampersaud ${ }^{1}$ noted that the anterior transclavicular approach is suited to access the tumor invading through the anterior to posterior components, such as the subclavian artery and the $\mathrm{T} 1$ vertebrae, which is the most challenging scenario in resecting the superior sulcus tumors.

\section{CONCLUSIONS}

The anterior transclavicular approach facilitates resection of lung cancer that invades the superior sulcus in the vicinity of the sternoclavicular joint, achieving adequate surgical margins. It is important to perform lobectomy through the second intercostal space anteriorly to minimize functional impairment of the shoulder.

\section{References}

1. de Perrot M, Rampersaud R. Surgical approaches to apical thoracic malignancies J Thorac Cardiovasc Surg. 2012;144:72-80,

2. Dartevelle PG, Chapelier AR, Macchiarini P, Lenot B, Cerrina J, Ladurie FL, et al. Anterior transcervical-thoracic approach for radical resection of lung tumors invading the thoracic inlet. J Thorac Cardiovasc Surg. 1993;105: 1025-34.

3. Grunenwald D, Spaggiari L. Transmanubrial osteomuscular sparing approach for apical chest tumors. Ann Thorac Surg. 1997;63:563-6.

4. de Perrot M, Rampersaud R. Anterior transclavicular approach to malignan tumors of the thoracic inlet: importance of the scapulothoracic articulation. J Thorac Cardiovasc Surg. 2007;134:801-3.

5. Dartevelle P, Mussot S. Anterior cervicothoracic approach to the superior sulcus for radical resection of lung tumor invading the thoracic inlet. Oper Tech Thorac Cardiovasc Surg. 2003;8:86-93. 